\title{
Transcriptome and gene expression analysis of docosahexaenoic acid producer Schizochytrium sp. under different oxygen supply conditions
}

\author{
Zhi-Qian Bi ${ }^{2}$, Lu-Jing Ren ${ }^{1,2^{*}}$, Xue-Chao Hu ${ }^{1,2}$, Xiao-Man Sun ${ }^{2}$, Si-Yu Zhu², Xiao-Jun Ji ${ }^{1,2}$ and He Huang ${ }^{1,3,4}$
}

\begin{abstract}
Background: Schizochytrium sp. is a promising strain for the production of docosahexaenoic acid (DHA)-rich oil and biodiesel, and has been widely used in the food additive and bioenergy industries. Oxygen is a particularly important environmental factor for cell growth and DHA synthesis. In general, higher oxygen supply favors lipid accumulation, but could lead to a reduction of the DHA percentage in total fatty acids in Schizochytrium sp. To tackle this problem, it is essential to understand the mechanisms regulating the response of Schizochytrium sp. to oxygen. In this study, we aimed to explore the acclimatization of this DHA producer to different oxygen supply conditions by examining the transcriptome changes.
\end{abstract}

Results: Two different fermentation processes, namely normal oxygen supply condition (shift agitation speeds from $400 \mathrm{rpm}$ to $300 \mathrm{rpm}$ ) and high oxygen supply condition (constant agitation speeds: $400 \mathrm{rpm}$ ), were designed to study how the fermentation characteristics of Schizochytrium sp. HX-308 were affected by different oxygen supply conditions. The results indicated that high oxygen supply condition resulted in $49 \%$ and $37.5 \%$ improvement in the maximum cell dry weight (CDW) and total lipid concentration, respectively. However, the DHA percentage in total fatty acids decreased to $35 \%$, which was $31.4 \%$ lower than that produced by normal oxygen supply condition. Moreover, transcriptome analysis was performed to explore the effect of the oxygen supply condition on genetic expression and metabolism. The results showed that glycolysis and pentose phosphate pathway metabolism-associated genes (hexokinase, phosphofructokinase, fructose-bisphosphate aldolase, glucose-6-phosphate dehydrogenase, and 6-phosphogluconate dehydrogenase) were substantially upregulated in response to high oxygen supply, resulting in more NADPH was available for Schizochytrium. Specially, high oxygen supply condition also led to genes ( $\Delta 6$ desaturase, $\triangle 12$ desaturase, FAS, ORFA, ORFB, and ORFC) involved in fatty acid biosynthesis upregulation. In addition, a transcriptional upregulation of catalase (CAT) became apparent under high oxygen supply condition, while superoxide dismutase (SOD) and ascorbate peroxidase (APX) were found to be down-regulated.

Conclusions: This study is the first to investigate the differences of gene expression at different levels of oxygen availability in the DHA producer Schizochytrium. The results of transcriptome analyses indicated that high oxygen supply condition resulting in more NADPH and acetyl-CoA production for cell growth and lipid synthesis in Schizochytrium. $\triangle 12$ desaturase and ORFC showed higher expression levels at high oxygen supply condition, which might

\footnotetext{
*Correspondence: renlujing@njtech.edu.cn

1 Jiangsu National Synergetic Innovation Center for Advanced Materials (SICAM), No. 30 South Puzhu Road, Nanjing 211816, People's Republic

of China

Full list of author information is available at the end of the article
} 
be the key regulators for enhancing fatty acid biosynthesis in the future. These results enrich the current knowledge regarding genetic expression and provide important information to enhance DHA production in Schizochytrium sp.

Keywords: Docosahexaenoic acid, Oxygen, Transcriptome, Fatty acids, NADPH

\section{Background}

Long-chain polyunsaturated fatty acids (PUFAs), especially omega-3 PUFAs, play an important role in alleviating cardiovascular diseases, hypertension, inflammation, and cancer $[1,2]$. Owing to their beneficial effects on human health, PUFAs have recently become the focus of intensive research. Fish oil is the traditional source of PUFAs, but this natural source has many disadvantages such as scarcity, unpleasant odor, and unstable quality, which are incompatible with the concept of healthy eating [3]. Therefore, ongoing biotechnology-based efforts are underway to develop and optimize alternative means for the production of high-quality PUFAs.

The marine strain of Schizochytrium sp. has a fast growth rate and high productivity, and it has been recognized as a great potential source for the production of PUFAs, particularly with regard to docosahexaenoic acid (DHA) [4]. In addition, Schizochytrium is promising production host for the sustainable generation of lipid-based bioproducts and bioenergy such as biodiesel [5, 6]. For instance, Johnson reported that Schizochytrium limacinum is a suitable feedstock for producing biodiesel via the direct transesterification method [7]. Since Schizochytrium is a heterotrophic aerobic microorganism, the oxygen supply has important effects on cell proliferation and lipid accumulation. It is reported that high oxygen level is favorable for cell growth, while DHA percentage in total fatty acids could be improved in oxygen-limited cultures [8-10]. With such a phenomenon, various fermentation strategies were developed by controlling oxygen supply to improve DHA productivity in Schizochytrium. Chi et al. [11] developed a two-stage control strategy for Schizochytrium limacinum SR21 in which they decreased dissolved oxygen (DO) level from 50 to $10 \%$ at $40 \mathrm{~h}$, and finally, the biomass and DHA yield were improved by $54.1 \%$ and $79.7 \%$, respectively. A two-stage oxygen supply control strategy based on the oxygen transfer coefficient $\left(\mathrm{K}_{\mathrm{L}} \mathrm{a}\right)$ was employed for Schizochytrium sp., whereby achieved dry cell weight of $92.7 \mathrm{~g} / \mathrm{L}$ and DHA concentration of $17.7 \mathrm{~g} / \mathrm{L}$, which improved by $25.3 \%$ and $63.88 \%$, respectively [12]. Similarly, Ren et al. [13] proposed a stepwise aeration controls strategy for Schizochytrium sp. HX-308. A 1.5-fold increase in the aeration rate at $24 \mathrm{~h}$ allowed the improvement of $5.97 \%$ and $7.98 \%$ in the cell dry weight and lipid concentration, respectively. These previous studies based on different oxygen supply control strategies improved cell growth and DHA production of Schizochytrium, and also provide guidance for large-scale industrial production. However, the potential effect of oxygen availability on the gene expression levels remains to be elucidated.

With the advancement of many new technologies, various omics analysis, such as genomics [14], lipidomics [15], proteomics [16], and metabolomics [17], have been considered a pivotal tool to help us understand molecular mechanism response to the changes of environment condition. It is reported that DHA is synthesized by a special polyketide synthase (PKS) system in Schizochytrium sp. [18]. Based on the information, Ye et al. [19] further used genome annotation data to reconstruct a genome-scale metabolic model, which could be used to elucidate the mechanism of DHA synthesize and predict the requirements of abundant acetyl-CoA and NADPH for DHA production. The potential effect of oxygen availability on the intracellular metabolite levels has been explored by comparative metabolomics analysis, and the results provided novel insights into the metabolomics characteristics during DHA production by Schizochytrium sp. [20]. In the meantime, large number of transcriptome studies in many oleaginous microorganisms, such as Aurantiochytrium [21], Yarrowia lipolytica [22], and Mortierella alpine [23], have been reported. However, for Schizochytrium sp., a relatively little was known about the variations of the primary transcriptome in response to the environment stress, and much efforts should be focus on discovering the molecular mechanisms by comparative transcriptome analysis.

In this study, to better understand the molecular basis of the observed effects of oxygen on DHA production, we here conducted an RNA-seq based transcriptomics analysis of Schizochytrium sp. HX-308 at different oxygen supply conditions. The analysis of global gene expression indicated that increased oxygen supply led to increased expression of, among others, genes related to NADPH and acetyl-CoA metabolism and ROS scavenging. This study deepens our scientific understanding of DHA production in Schizochytrium sp. and provides an important new functional genomics information resource that will help to generate testable hypotheses about, and engineering strategies based on, the molecular mechanisms underlying both lipid accumulation and DHA production by this industrially important marine fungus. 


\section{Results}

Effects of oxygen supply on the fermentation performance of Schizochytrium sp.

Two different fermentation processes, namely normal oxygen supply condition (shift agitation speeds from $400 \mathrm{rpm}$ to $300 \mathrm{rpm}$ ) and high oxygen supply condition (constant agitation speeds: $400 \mathrm{rpm}$ ), were designed to study the effects of different oxygen supply on the fermentation performance of Schizochytrium sp. Essentially, the value of volumetric oxygen-transfer coefficient $\left(\mathrm{K}_{\mathrm{L}} \mathrm{a}\right)$ directly determined availability of oxygen in culture medium and high $\mathrm{K}_{\mathrm{L}}$ a forced more oxygen to be dissolved in the fermentation broth [9]. The value of $K_{L}$ a at $300 \mathrm{rpm}$ was only $75.2 \mathrm{~h}^{-1}$, while, at $400 \mathrm{rpm}$, the value reached $136.8 \mathrm{~h}^{-1}$. Therefore, the two agitation speeds represented different oxygen supply conditions. Figure 1 exhibits the effects of the oxygen supply conditions on cell growth, glucose consumption, and lipid concentration of Schizochytrium sp. HX-308 (F1: normal oxygen supply condition; F2: high oxygen supply condition). Compared with normal oxygen supply condition, high oxygen supply could accelerate cell growth and glucose consumption, leading to a shortening fermentation period. As shown in Fig. 1a, cell dry weight (CDW) sharply increased and reached to $107 \mathrm{~g} / \mathrm{L}$ at $84 \mathrm{~h}$ in F2, which was $49 \%$ higher than that of F1. However, it was not observed until $132 \mathrm{~h}$ that the CDW in F1 reached to a maximum $(104 \mathrm{~g} / \mathrm{L})$. Cells in F2 consumed more glucose, especially in the first $90 \mathrm{~h}$. The glucose consumption of F2 was $361 \mathrm{~g} / \mathrm{L}$ at $84 \mathrm{~h}$, while this value in F1 was only $161 \mathrm{~g} / \mathrm{L}$ (Fig. 1b). This result was also similar
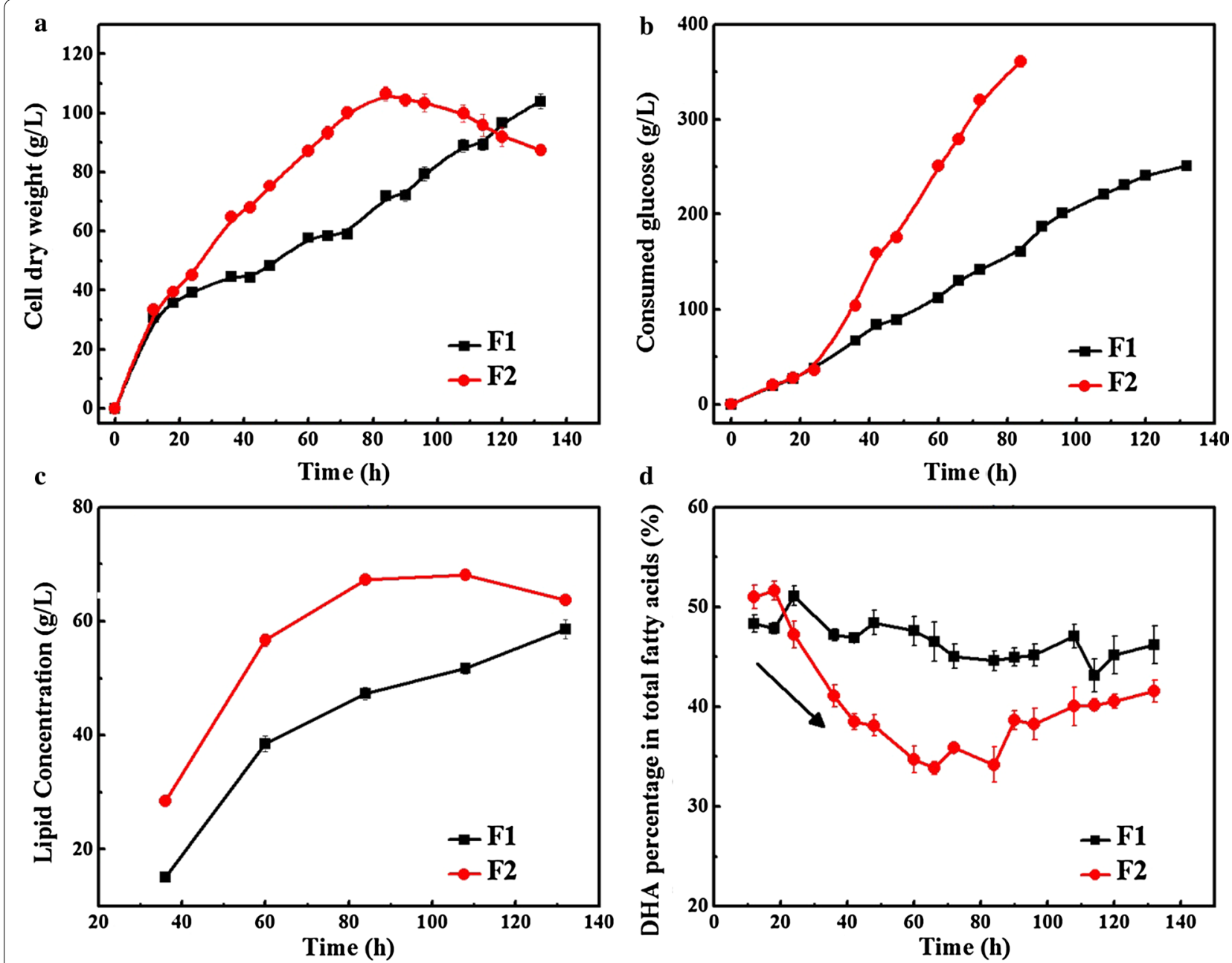

Fig. 1 Fermentation characteristic of Schizochytrium sp. HX-308 under different oxygen supply conditions. a Cell dry weight (g/L), b glucose consumption ( $\mathrm{g} / \mathrm{L})$, c lipid yield ( $\mathrm{g} / \mathrm{L})$, d DHA percentage in total fatty acids (\%). F1, normal oxygen supply condition; F2, high oxygen supply condition 
to a previous study that high oxygen supply condition could improve the substrate utilization capacity [18]. Similarly, lipid concentration also increased with the increment of cell dry weight in two fermentation processes. At the same time point of $84 \mathrm{~h}$, lipid concentration in F2 and F1 was $67.31 \mathrm{~g} / \mathrm{L}$ and $47.34 \mathrm{~g} / \mathrm{L}$, respectively. Lipid concentration in F2 is increased by $42.19 \%$ than $\mathrm{F} 1$. In addition, the maximum lipid concentration of $58.66 \mathrm{~g} / \mathrm{L}$ in $\mathrm{F} 1$ consumed $132 \mathrm{~h}$, while the same lipid concentration in F2 less than $70 \mathrm{~h}$, saving $47 \%$ in fermentation time (Fig. 1c).

Moreover, a significant difference was observed in the fatty acid composition of Schizochytrium sp. HX-308 in response to different oxygen supply conditions (Table 1). Saturated fatty acids (SFAs) are mainly composed of C14:0 and C16:0 in Schizochytrium, while PUFAs mainly consist of eicosapentaenoic acid (EPA), docosapentaenoic acid (DPA), and DHA. SFA percentage in total fatty acids increased over time from $29.59 \%$ at $24 \mathrm{~h}$ to $34.64 \%$ at $72 \mathrm{~h}$ in F2. However, only $24.03 \%$ SFAs was observed at $72 \mathrm{~h}$ in $\mathrm{F} 1$, which was $30.63 \%$ lower than that of F2. Meanwhile, high oxygen condition led to less PUFAs accumulation. The final PUFAs percentage in total fatty acids was only $58.19 \%$, which represented $15.6 \%$ decreased over the normal oxygen supply. Most importantly, the DHA percentage in total fatty acids of different oxygen availability conditions showed differences. As shown in Fig. 1d, DHA percentage in total fatty acids was reached a peak value of $51.66 \%$ at $18 \mathrm{~h}$, but decreased sharply to $34.73 \%$ at $60 \mathrm{~h}$. Later, it slightly increased and finally remained at $41 \%$ until the end of fermentation. Notably, this phenomenon was not observed in F1. DHA percentage in TFAs at F1 always remained constant at $45-50 \%$ throughout the fermentation and, finally, reached to $46.20 \%$, which was $11.11 \%$ higher than that of F2. These results indicated that the shift of string speed might play an important role in maintaining the constant DHA percentage in total fatty acids. Deciphering the mechanism is quiet important to understand the biosynthesis of fatty acids in Schizochytrium sp.

\section{Illumina HiSeq mRNA sequencing and functional} classification of unigenes

To obtain an overview of the Schizochytrium sp. HX-308 gene expression profiles at different oxygen supply conditions, we carried out the RNA-seq analysis. In total, 47.51 million reads and 17,778 transcripts were generated from two libraries. All the original reads have been deposited in the NCBI Sequence Read Archive (http://trace.ncbi. nlm.nih.gov/Traces/sra/, Accession No. SRP156360). The length distribution of transcripts can be seen in Additional file 1: Figure S1. Moreover, 8911(normal oxygen supply condition) and 8867 (high oxygen supply condition) genes were identified by aligning clean reads against the reference genome of Schizochytrium. HX-308 [24]. Among these genes, the number of co-expressed gene in both normal and high oxygen supply conditions was 8796 (97.9\%). To further investigate the gene expression profiles of the 8796 co-existed genes, differences in gene expression at different oxygen supply conditions were examined. A total of 890 unigenes with obvious different expression levels were identified. Among these 890 differentially expression genes (DEGs) shown in Additional file 1: Figure S1C, there were 307 upregulated and 583 down-regulated between normal and high oxygen supply conditions. To obtain their functional information, these DEGs were annotated against GO database. As shown in Additional file 1: Figure S2, the majority of DEGs were classified as belonging to the category biological process $(941 ; 43.86 \%)$, followed by cellular component $(473 ; 22.05 \%)$ and molecular function $(731 ; 34.08 \%)$. In detail, "cellular process" $(259 ; 12.07 \%)$ and "metabolic process" (289; 13.47\%), "cell" (101; 4.73\%) and "cell part" (101; 4.73\%), "binding" (325; 15.15\%), and "catalytic activity" (293; 13.66\%), all ranked among the top classes in the three levels of GO ontology.

To understand the biological function of the identified DEGs, 890 genes were all mapped to the related pathways in the KEGG database and chose a $q$ value of $\leqq 0.05$ as significant among the DEGs. As shown in Additional file 1: Figure S3, these DEGs were mapped to the five most significantly enriched pathways $(\mathrm{q} \leqq 0.025)$, including

Table 1 Differences of the fatty acid composition of Schizochytrium sp. HX-308 in response to different oxygen supply conditions

\begin{tabular}{|c|c|c|c|c|c|c|c|c|c|c|}
\hline \multirow[t]{2}{*}{ Time/h } & \multicolumn{5}{|c|}{ Percent of fatty acids in normal oxygen supply (\%) } & \multicolumn{5}{|c|}{ Percent of fatty acids in high oxygen supply (\%) } \\
\hline & C14:0 & C16:0 & EPA & DPA & DHA & C14:0 & C16:0 & EPA & DPA & DHA \\
\hline 24 & $5.21 \pm 0.09$ & $19.35 \pm 0.59$ & $1.11 \pm 0.05$ & $15.29 \pm 0.46$ & $51.95 \pm 1.15$ & $6.24 \pm 0.13$ & $23.35 \pm 0.69$ & $1.0 \pm 0.03$ & $13.78 \pm 0.45$ & $47.35 \pm 0.64$ \\
\hline 48 & $6.66 \pm 0.15$ & $20.05 \pm 0.61$ & $1.07 \pm 0.03$ & $17.45 \pm 0.57$ & $47.69 \pm 0.83$ & $8.99 \pm 0.21$ & $25.71 \pm 0.72$ & $0.89 \pm 0.01$ & $15.61 \pm 0.48$ & $38.27 \pm 0.61$ \\
\hline 72 & $5.72 \pm 0.09$ & $18.31 \pm 0.43$ & $1.59 \pm 0.04$ & $18.13 \pm 0.63$ & $47.88 \pm 0.59$ & $10.33 \pm 0.23$ & $24.31 \pm 0.56$ & $1.01 \pm 0.03$ & $14.52 \pm 0.50$ & $36.18 \pm 0.86$ \\
\hline 96 & $6.01 \pm 0.13$ & $17.21 \pm 0.42$ & $1.88 \pm 0.05$ & $19.62 \pm 0.64$ & $45.95 \pm 0.76$ & $11.46 \pm 0.2$ & $21.54 \pm 0.65$ & $1.20 \pm 0.04$ & $16.49 \pm 0.59$ & $39.8 \pm 0.59$ \\
\hline 120 & $7.79 \pm 0.13$ & $15.14 \pm 0.39$ & $1.49 \pm 0.05$ & $20.9 \pm 0.67$ & $46.59 \pm 0.63$ & $9.96 \pm 0.15$ & $20.74 \pm 0.58$ & $1.22 \pm 0.04$ & $16.39 \pm 0.61$ & $40.58 \pm 0.46$ \\
\hline
\end{tabular}

Data were means $\pm S D$ of three independent experimental replicates 
ABC transporters, amino sugar, and nucleotide sugar metabolism, biosynthesis of secondary metabolites, fatty acid metabolism, and glycolysis/gluconeogenesis. Interestingly, the DEGs involved in the significantly enriched pathways were directly associated with the energy metabolism. For example, ABC transporters use the ATP to transport diverse substrates [19]; glycolysis could generate NADH and ATP for cell growth.

\section{Differential gene expression related to NADPH and acetyl-CoA}

For oleaginous microorganism, acetyl-CoA and NADPH are two important precursors for lipid accumulation. In this study, based on the gene annotation, we paid particularly attention to differentially expressed genes related to the supply of acetyl-CoA and NADPH. Table 2 compares the transcript abundances of the genes encoding enzymes involved the central carbon metabolism pathways, including glycolysis (EMP), pentose phosphate pathway (PPP) and tricarboxylic acid (TCA) cycle. Compared with normal oxygen supply condition, the hexokinase (HK) and phosphofructokinase (PFK), which participate in glycolytic pathway, were upregulated at high oxygen supply condition by 3.42 - and 2.81 -fold, respectively. Moreover, the expression of fructose-bisphosphate aldolase (FBA) which was also involved in glycolysis was obviously increased by more than 7.20 -fold. In addition, three genes encoding pyruvate kinase (PK) were detected with different expression changes, and two genes (ID: A0923 and A4432) were slightly downregulated, while another one (ID: A5006) was obviously upregulated by 3.18 -fold. In addition, various enzymes involved in the PPP, including glucose-6-phosphate dehydrogenase (G6PD), 6-phosphogluconate dehydrogenase (6PGD), ribulose-phosphate-3-epimerase, and ribose 5-phosphate isomerase A, were upregulated. The higher expression of genes indicated that sugar uptake and its catabolism were constrained due probably to oxygen limiting at normal oxygen condition. In addition, citrate synthase (CS) which participate in TCA cycle was also upregulated 1.71-fold in the high oxygen supply condition. We can also see that the expression of isocitrate dehydrogenase (IDH) kept at

Table 2 Differential expression of key genes related to NADPH and acetyl-CoA

\begin{tabular}{|c|c|c|c|c|c|}
\hline \multirow[t]{2}{*}{ Pathway } & \multirow[t]{2}{*}{ Enzyme } & \multirow[t]{2}{*}{ Gene ID } & \multicolumn{2}{|l|}{ RPKM } & \multirow{2}{*}{$\begin{array}{l}\text { Up/ } \\
\text { downregulated }\end{array}$} \\
\hline & & & Normal & High-oxygen & \\
\hline \multirow[t]{6}{*}{ EMP } & HK & SchizochytriumA2194 & 182.23 & 623.48 & $3.42 \uparrow$ \\
\hline & PFK & SchizochytriumA2542 & 181.29 & 510.14 & $2.81 \uparrow$ \\
\hline & FBA & SchizochytriumA3279 & 912.09 & 6566.81 & $7.20 \uparrow$ \\
\hline & PK & SchizochytriumA5008 & 360.54 & 1144.91 & $3.18 \uparrow$ \\
\hline & & SchizochytriumA0923 & 86.38 & 73.29 & $1.18 \downarrow$ \\
\hline & & SchizochytriumA4432 & 73.81 & 53.34 & $1.38 \downarrow$ \\
\hline \multirow[t]{4}{*}{ PPP } & G6PD & SchizochytriumA4008 & 219.6 & 418.4 & $1.91 \uparrow$ \\
\hline & 6PGD & SchizochytriumA3341 & 553.18 & 778.82 & $1.41 \uparrow$ \\
\hline & RPE & SchizochytriumA1080 & 81.89 & 86.24 & $1.05 \uparrow$ \\
\hline & RPIA & SchizochytriumA0514 & 43.28 & 99.67 & $2.30 \uparrow$ \\
\hline \multirow[t]{7}{*}{ TCA } & CS & SchizochytriumA5632 & 483.05 & 825.88 & $1.71 \uparrow$ \\
\hline & $\mathrm{IDH}$ & SchizochytriumA8242 & 280.02 & 282.25 & $1.00 \uparrow$ \\
\hline & KGDC & SchizochytriumA6065 & 7.6 & 4.7 & $1.62 \downarrow$ \\
\hline & & SchizochytriumA6274 & 387.51 & 349.09 & $1.11 \downarrow$ \\
\hline & & SchizochytriumA8288 & 249.99 & 237.49 & $1.05 \downarrow$ \\
\hline & ME & SchizochytriumA9142 & 799.4 & 907.84 & $1.14 \uparrow$ \\
\hline & $\mathrm{ACL}$ & SchizochytriumA2237 & 63.24 & 67.27 & $1.06 \uparrow$ \\
\hline \multirow[t]{5}{*}{ Peroxisome } & SOD & SchizochytriumA5508 & 123.65 & 61.05 & $2.03 \downarrow$ \\
\hline & & SchizochytriumA4372 & 622.26 & 500.95 & $1.24 \downarrow$ \\
\hline & & SchizochytriumA0375 & 1102.97 & 1083.19 & $1.02 \downarrow$ \\
\hline & CAT & SchizochytriumA4694 & 45.8 & 70.63 & $1.54 \uparrow$ \\
\hline & APX & SchizochytriumA2136 & 700.57 & 395.35 & $1.77 \downarrow$ \\
\hline
\end{tabular}

RPKM The percentage of a gene covered by reads. The RPKM method is able to eliminate the influence of different gene length and sequencing discrepancy on the calculation of gene expression. Therefore, the calculated gene expression can be directly used for comparing the difference of gene expression among samples. Numbers of up/down-regulated means RPKM high oxygen supply $/$ RPKM $_{\text {normal oxygen supply }}{ }^{*}$ The up arrow indicated that gene was upregulated under high oxygen supply condition. The down arrow indicted that gene was down-regulated under high oxygen supply condition 
a constant level in both conditions. In contrast, high oxygen supply condition led to three genes, which have been annotated as coding for $\alpha$-oxoglutarate dehydrogenase in Schizochytrium sp., which represented a consistent upregulation trend. Furthermore, malic enzyme (ME) and ATP citrate lyase (ACL), major providers of $\mathrm{NADPH}$, were also slightly upregulated.

\section{Differential gene expression related to antioxidant defense system}

Antioxidant defense system plays an important role in counteracting the various types of oxidative stress [25]. In this study, we detected three kinds of antioxidant enzymes, including superoxide dismutase (SOD), ascorbate peroxidase (APX), and catalase (CAT). The transcription results indicated that two genes (ID: A4372 and A0375) encoding SOD showed no significant change under high oxygen supply condition, while another gene (ID: A5508) was obviously upregulated by 2.03 -fold. We also found the expression of APX decreased nearly 1.77 -fold, suggesting that less APX was needed to deal with oxidative stress in cells under high oxygen condition. In contrast, high oxygen supply condition led to the CAT upregulation, which was mainly responsible for the disposal of free circulating $\mathrm{H}_{2} \mathrm{O}_{2}$. The inconsistency of antioxidant enzymes expression under different oxygen supply conditions indicated that different mechanism against oxidative stress exist in the peroxisomes, which may be related to the deactivation of hydrogen peroxide. In addition, four genes encoding glutathione peroxidase (GSH-Px) were detected, which was regarded as a major protective system against endogenously and exogenously induced lipid peroxidation. When Schizochytrium was cultured with high oxygen supply condition, these genes were all upregulated by 1.37-, 1.08-, 1.16-, and 1.17-fold, respectively.

\section{Differential gene expression related to fatty acid biosynthesis}

To investigate the mechanism that contribute to the change of DHA percentage in total fatty acids at different oxygen supply conditions in Schizochytrium sp. HX-308, the differentially expressed genes involving the pathway of fatty acid biosynthesis were analyzed. As shown in Table 3, two $\Delta 6$ desaturases were detected, and the expression of these genes increased 6.9 and 1.87 times under high oxygen condition, respectively. By contrast, three $\Delta 8$ desaturases showed no significant changes under different oxygen availability conditions. Remarkably, the $\Delta 12$ desaturase that catalyzes the conversion of oleic acid (C18:1) into linoleic acid (C18:2) showed superior performance to the other desaturases. The RPKM value which represents the transcription abundance of the gene reached 7076 in high oxygen supply condition. These data indicated that the $\Delta 12$ desaturase was significant for polyunsaturated fatty acid synthesis through the desaturase/ elongase pathway in Schizochytrium sp. Unsurprisingly, the high oxygen supply condition led to FAS gene was obviously upregulated by nearly 4.0 -fold. Moreover, we identified three genes associated with the PKS route of PUFAs biosynthesis-ORFA, ORFB, and ORFC. During the cell growth stage, all genes involved in the PKS pathway showed high expression levels [26]. Our transcription results showed that PKS genes (ORFA, ORFB, and ORFC), which are directly involved in the DHA biosynthesis, were significantly differentially expressed between normal and high oxygen supply conditions. With high oxygen supply condition, ORFA, ORFB, and ORFC were upregulated 1.88-, 1.75-, and 2.25-fold, respectively. In addition, we can see that ORFA and ORFC had a higher expression levels with RPKM values reached to 1851.4 and 1821.4, which indicated that they played more important roles in lipid synthesis, especially under high oxygen supply condition.

\section{Verification of gene expression through qPCR}

To validate the transcriptome data, qPCR was used to verify the RNA-seq results for six genes (FAS, ORFA, ORFB, ORFC, ACC, and ME), most of which were

Table 3 Differential expression of key genes related to fatty acid biosynthesis

\begin{tabular}{|c|c|c|c|c|c|c|c|c|c|c|c|}
\hline \multirow[t]{2}{*}{ Gene } & \multicolumn{11}{|c|}{ Fatty acid biosynthesis } \\
\hline & \multicolumn{3}{|c|}{$\Delta$-8 desaturase } & \multicolumn{2}{|c|}{$\Delta-6$ desaturase } & \multirow[t]{2}{*}{$\Delta-12$ desaturase } & \multirow[t]{2}{*}{ Elongase } & \multirow[t]{2}{*}{ FAS } & \multirow[t]{2}{*}{ ORFA } & \multirow[t]{2}{*}{ ORFB } & \multirow[t]{2}{*}{ ORFC } \\
\hline RPKM & & & & & & & & & & & \\
\hline Normal-oxygen & 161.4 & 0.9 & 50.56 & 1.45 & 4.8 & 2822.5 & 199.96 & 167.98 & 987.24 & 348.6 & 591.44 \\
\hline High-oxygen & 142.5 & 1.96 & 46.69 & 10.02 & 8.98 & 7046.7 & 128.16 & 666.83 & 1851.4 & 610.65 & 1328.4 \\
\hline
\end{tabular}

The RPKM method is able to eliminate the influence of different gene length and sequencing discrepancy on the calculation of gene expression. Therefore, the calculated gene expression can be directly used for comparing the difference of gene expression among samples 
associated with the PKS pathway of fatty acid biosynthesis. The results showed that the expression levels of most genes were consisted with those acquired by transcriptome analysis, indicating that the RNA-seq data are reliable (Additional file 1: Figure S4).

\section{Discussion}

As is well known, oxygen supply is a crucial factor for cell growth and lipid accumulation in Schizochytrium sp. In recent years, we focused on investigating the influence of oxygen on DHA production and developed diverse oxygen control strategies to increase DHA production [2729]. However, the specific expression of genes involved in the cell response to oxygen has not yet been explored at the transcriptional level for Schizochytrium sp. Based on next-generation sequencing techniques, we first analyzed the transcriptome differences and revealed various genes that were differentially expressed between normal and high oxygen supply conditions in Schizochytrium sp. (all the genes mentioned in this paper were listed in Additional file 1: Table S1). These results provide new insights into the effects of oxygen on fatty acid biosynthesis and accumulation in Schizochytrium sp. at the transcriptomic level.

The key to fatty acid biosynthesis is a sufficient supply of NADPH and acetyl-CoA. In most microorganisms, glucose-6-phosphate dehydrogenase (G6PD; EC1.1.1.49), 6-phosphogluconate dehydrogenase (6PGD; EC1.1.1.44), malic enzyme (ME; EC1.1.1.40), and isocitrate dehydrogenase (IDH; EC 1.1.1.42) are recognized as principally responsible for generating NADPH (Fig. 2a). G6PD and 6PGD are the key enzymes of the pentose phosphate pathway. Many studies have reported that different adverse environments, including cold stress [30], oxidative stress [31], and high-salinity stress [32], induce a high activity of G6PD and 6PGD to maintain the abundant supply of NADPH. In addition, Osada et al. [33] demonstrated that overexpression of G6PD and 6PGD in the oleaginous diatom Fistulifera solaris accelerated the lipid accumulation by increased NADPH production. Our transcriptome data showed that when Schizochytrium sp. HX-308 was cultured at high oxygen supply condition, G6PD and 6PGD were upregulated by 1.91 -fold and 1.41-fold, respectively. Other enzymes involved in the PPP, such as ribulose-phosphate-3-epimerase and ribose 5-phosphate isomerase $\mathrm{A}$, were also upregulated, so we speculated that the PPP was enhanced to some extent and generated much more NADPH under high oxygen condition. Traditionally,ME was considered to be the major provider of NADPH for fatty acid synthesis in oleaginous microbes, which catalyzes the reversible oxidative decarboxylation of malate and is a link between the glycolytic pathway and the citric acid cycle [34-36]. However, these
NADP-reducing enzyme responses vary under different environments. For example, the transcription of ME showed no significant difference by oxidative stress, whereas nitrogen starvation could enhanced ME expression in Drosophila melanogaster [37]. Our data showed that high oxygen supply condition resulted in ME expression upregulated 1.14-fold, but whether upregulated ME could provide more NADPH for lipid accumulation need to be investigated. Interestingly, IDH, which catalyzes the oxidative decarboxylation of isocitrate to 2-oxoglutarate showed no differential expression under different oxygen supply conditions. Similar results were reported that IDH expression showed no significant change under cold and oxidative stresses $[21,38]$. In addition to NADPH, acetyl-CoA is a very hot topic in the lipid research field as an indispensable precursor for fatty acid biosynthesis [39]. Various catabolic reactions, including glycolysis, $\beta$-oxidation, and the catabolism of branched amino acids could produce acetyl-CoA to supply energy for cell [40, 41]. It has been reported that only oleaginous microorganisms can produce acetyl-CoA via citric acid cleavage [36]. Thus, ATP citrate lyase, which catalyzes the cleavage of citrate, has been recognized as a major source of acetyl-CoA for fatty acid biosynthesis. Our study found that ACL expression was less susceptible to the effects of oxygen supply, and its transcript abundance did not change under different oxygen availability conditions. By contrast, many genes involved in the glycolysis showed significantly change induction by supply conditions. When Schizochytrium was cultured at high oxygen supply condition, HK that catalyzes phosphorylating glucose into glucose-6-phosphate was upregulated by 3.4-fold. Similarly, Chen et al. [42] found glycerol as carbon source could enhance the expression of HK in Schizochytrium. As one of key enzymes in the glycolysis, PFK, is responsible for catalyzing the ATP-dependent phosphorylation of fructose-6-phosphate to form fructose 1, 6-bisphosphate. We found the expression of PFK transcripts increased by 2.81 -fold under high oxygen supply condition. Cheng et al. [32] obtained similar results in Nitzschia sp. under high-salinity stress condition. Unexpectedly, we found that FBA showed higher sensitive to the change of oxygen supply condition in the glycolysis, whereby the expression of FBA was upregulated by more than 7.2fold. Different enzymatic changes indicated that complex mechanisms that regulate NADPH and acetyl-CoA generation exist in Schizochytrium, which may be related to various metabolism activities of cells, such as lipids synthesized and the other components of biomass synthesized need reducing power, acetyl-CoA and other factors. Considering the expression of genes related to the PPP and glycolysis increased by varying degrees, the production of NADPH and acetyl-CoA were probably improved 


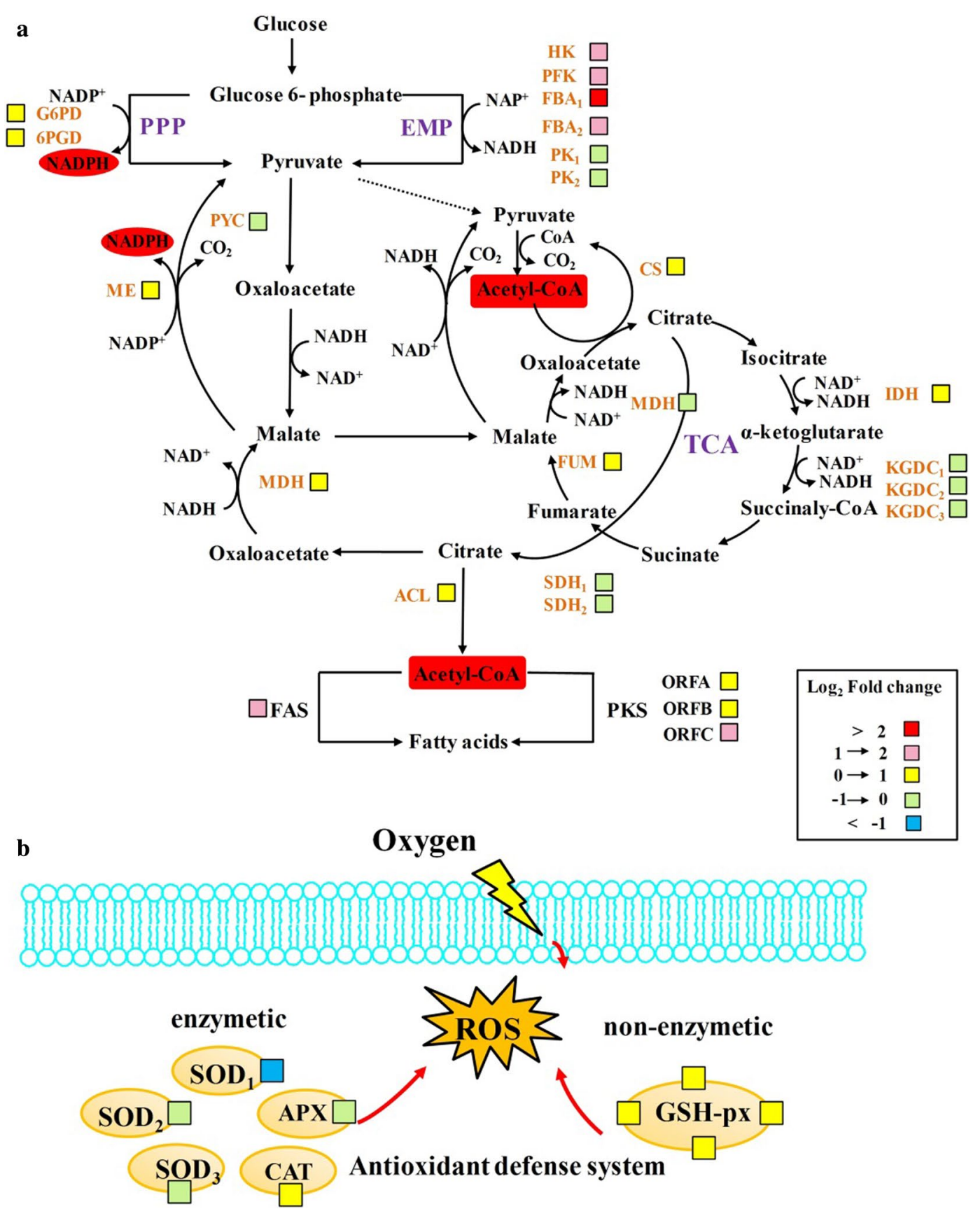

Fig. 2 Changes in transcript abundance of genes involved in central metabolic pathways and antioxidant system when Schizochytrium sp. HX-308 was cultured with high oxygen supply condition. a Transcriptional regulation of the central carbon metabolic pathways, including glycolysis, pentose phosphate pathway, and tricarboxylic acid cycle. b Transcriptional regulation of the antioxidant defense system. Key enzymes are included in the map and presented as their names. Gene fold changes as indicated by color boxes. Glycolysis; PPP, pentose phosphate pathway; G6PD, glucose-6-phosphate dehydrogenase; 6PGD, 6-phosphogluconate dehydrogenase; EMP, Embden-Meyerhof-Parnas pathway; HK, hexokinase; PFK, phosphofructokinase; FBA, fructose-bisphosphate aldolase; PYC, pyruvate carboxylase; PK, pyruvate kinase; TCA, tricarboxylic acid; CS, citrate synthase; $\mathrm{MDH}$, malate dehydrogenase; FUM, fumarate hydratase; IDH, isocitrate dehydrogenase; ME, malic enzyme; $A C L$, citrate lyase; KGDC, 2-oxoglutarate dehydrogenase; SDH, succinate dehydrogenase; SOD, superoxide dismutase; CAT, catalase; APX, ascorbate peroxidase; ROS, reactive oxygen species; GSH-Px, glutathione peroxidase 
in Schizochytrium sp. HX-308 under condition of high oxygen supply.

There are two known metabolic pathways for fatty acid biosynthesis in Schizochytrium sp., a fatty acid synthase (FAS) pathway, and a polyunsaturated fatty acid synthase pathway (PKS) [18]. In the FAS route, saturated fatty acids (e.g., C14:0 and C16:0) are produced by the complex FAS enzyme, after which they are modified through successive desaturation and elongations steps, resulting in the synthesis of various PUFAs [43]. However, this standard desaturation-elongation pathway is incomplete in Schizochytrium, whereby the activity of specific desaturase and elongase were not detected [18]. Lippmeier et al. [44] reported $\Delta 5, \Delta 6$, and $\Delta 9$ elongases' activities in Schizochytrium sp. ATCC20888, but they did not find $\Delta 12$ desaturation activity. Hoang et al. [45] identified only two unigenes encoding elongase protein and one unigene encoding $\Delta 6$ desaturase. In our study, three kinds of desaturases were identified: the $\Delta 6, \Delta 8$, and $\Delta 12$ desaturases. Under high oxygen supply condition, $\Delta 6$ and $\Delta 8$ desaturases showed no obviously changes, but the gene encoding $\Delta 12$ desaturase was significantly upregulated, more than 2.5 -fold. Similarly, high oxygen enhanced the expression of $\Delta 12$ desaturase in Acanthamoeba castellanii, resulting in the enzyme's activity upregulated by tenfold [44]. As $\Delta 12$ desaturase catalyzes C18:1 to C18:2, we assumed that more $C 18: 2$ would be generated by $\Delta-12$ desaturase in Schizochytrium under high oxygen supply condition and further experiments are needed to verify this idea. However, compared with $\Delta 12$ desaturase, the expression of $\Delta 6$ desaturase and $\Delta 8$ desaturase was relatively low, which indicated that these two enzymes played little role in the fatty acid synthesis. Chen et al. [42] suggested that, when glycerol was the carbon source, the FAS gene upregulation led to a higher DHA concentration in Schizochytrium sp. S056. Our data showed that with a high oxygen supply condition, FAS gene also presented a higher expression level, which is in line with the fact that FAS pathway process requires the participation of molecular oxygen. The higher expression of genes related to FAS pathway might be a reason why high oxygen supply condition led to the increase of saturated fatty acids percentage in total fatty acids. Different from FAS pathway, PKS pathway does not involve the typical desaturation-elongation steps and it does not require molecular oxygen [18]. In Schizochytrium, PKS pathway just utilizes the PKS gene cluster mechanism to de novo synthesize PUFAs from acyl-CoA. Our study found that a high oxygen supply was not beneficial for PUFAs accumulation. When Schizochytrium was cultivated with high oxygen supply condition, the PUFAs' (EPA, DPA, and DHA) percentage in total fatty acids was only $58.19 \%$, which is $15.6 \%$ lower than normal oxygen supply condition. The transcriptome data showed that the expression of genes (ORFA, ORFB, and ORFC) involved in the PKS pathway was increased under high oxygen supply condition. Especially, high oxygen supply condition led to the ORFC which contains two DH (dehydrase) domains and one ER (enoyl-ACP reductase) upregulated by 2.25 -fold. AcetylCoA carboxylase (ACC), which produces malonyl-CoA for use in both the FAS and PKS pathways, is considered as a key enzyme in the fatty acid biosynthesis [46]. Ma et al. [21] found that, in Aurantiochytrium, ACC was upregulated and provided more malonyl-CoA under cold stress. We observed high oxygen supply condition also enhanced the expression of ACC in Schizochytrium. Compared with normal oxygen supply condition, when Schizochytrium was cultivated at high oxygen supply condition, ACC was upregulated by 2.80 -fold, suggesting that more malonyl-CoA was produced for lipid synthesis.

It is accepted that a higher oxygen supply is beneficial for cell growth and lipid accumulation. However, in an oxygen-rich environment, lipids, and among them especially the polyunsaturated fatty acids could be oxidized [47]. Johansson [48] found that lipid peroxidation could accumulate reactive oxygen species (ROS) and cause damage to cells. In fact, for oleaginous microorganisms such as Schizochytrium, despite the fact that most oxygen consumed is used to provide energy for cell growth and lipid accumulation, there always is an inevitable aspect that of its conversion into ROS [49]. To cope with this oxidative stress, cells could activate their detoxification machinery, which consists of enzymatic as well as non-enzymatic defense systems (Fig. 2b). Superoxide dismutases (SOD), which catalyze the dismutation of the superoxide anion $\mathrm{O}_{2}{ }^{-}$into $\mathrm{H}_{2} \mathrm{O}$ and $\mathrm{O}_{2}$, are considered as the first line of defense against ROS. Tripathi et al. $[50,51]$ found that SOD activity was enhanced in Ditylum brightwellii and Scenedesmus sp. in response to copper stress. Likewise, Kumar et al. [52] observed that SOD activity was significantly increased when microorganism was exposed to high-salinity stress. In this study, we detected three SOD and they were upregulated by varying degrees in Schizochytrium under high oxygen supply condition. Further research need to be explored the exact implication of each individual SOD in response to environment change. Catalases play a central role in defense against oxidative stress, which catalyze the breakdown of $\mathrm{H}_{2} \mathrm{O}_{2}$ into $\mathrm{O}_{2}$ and $\mathrm{H}_{2} \mathrm{O}$. We found that a high oxygen supply condition led to the CAT upregulation in Schizochytrium, suggesting that more hydrogen peroxide was produced. Not only lipids' oxidation but also other biochemical processes are also demanding antioxidant enzymes, which might affect gene expression level. In addition, non-enzymatic defense systems like glutathione can counteract the 
various types of oxidative stress. For instance, $\mathrm{H}_{2} \mathrm{O}_{2}$ is reduced by a glutathione peroxidase using GSH as a reductant [53]. In this study, we found four kinds of glutathione peroxidases that were upregulated under high oxygen supply condition. We speculated that, under high oxygen supply condition, more lipids were oxidized and thus more hydrogen peroxide was formed increasing the need of glutathione peroxidase.

\section{Conclusions}

In this study, we controlled oxygen supply conditions by altering agitation speeds and conducted a transcriptome analysis to explore the changes of gene expression between normal-oxygen supply and high oxygen supply samples. The explorative analysis of transcriptional profiles was focused on the central carbon metabolism, fatty acids synthesis, and oxidative stress. The results indicated that many genes involved in the metabolism of acetyl-CoA and NADPH were preferentially expressed in the high oxygen supply condition. This work helps us get a series of differential expression genes involved in central metabolic pathways and fatty acid biosynthesis under different oxygen supply conditions, which provide important genomics information that will help to enhance DHA production in Schizochytrium sp. by engineering strategies in the future.

\section{Methods}

\section{Microorganism and culture conditions}

Schizochytrium sp. HX-308 (CCTCC M209059), isolated from seawater and preserved in the China Center for Type Culture Collection (CCTCC), was used in this study. This strain was preserved in $20 \%$ (v/v) glycerol at $-80{ }^{\circ} \mathrm{C}$. The seed culture medium and conditions were the same as those used in our previous study [20]. The culture preserved in the glycerine tube was inoculated into a $500-\mathrm{mL}$ shake flask containing $100-\mathrm{mL}$ medium and cultivated for $24 \mathrm{~h}$ at $28{ }^{\circ} \mathrm{C}$. After culturing for three generations, the seed cultures $(10 \% \mathrm{v} / \mathrm{v})$ were transferred to two 5 - $\mathrm{L}$ bioreactors with a working volume of $3 \mathrm{~L}$. The agitation speeds were both set up $400 \mathrm{rpm}$ before $18 \mathrm{~h}$. After that, one fermentor maintained agitation speed to $400 \mathrm{rpm}\left(\mathrm{K}_{\mathrm{L}} \mathrm{a}: 136.8 \mathrm{~h}^{-1}\right.$, namely high oxygen supply condition), another fermentor set agitation speed to $300 \mathrm{rpm}\left(\mathrm{K}_{\mathrm{L}} \mathrm{a}: 136.8 \mathrm{~h}^{-1}\right.$, namely normal oxygen supply condition). The aeration was controlled at $0.18 \mathrm{~m}^{3} / \mathrm{h}$ to achieve the aeration rate of 1 volume of air per volume of liquid per minute (vvm). All fermentations were inoculated with the same culture and conducted at free $\mathrm{pH}$.
Cell dry weight, glucose, total lipids, fatty acids analysis, and oxygen transfer coefficient detection

Cell dry weight was determined gravimetrically by filtering 10-mL fermentation broth using centrifuge for $5 \mathrm{~min}$ at $4500 \mathrm{~g}$. The cells were subsequently transferred to a weighed filter paper and dried at $60{ }^{\circ} \mathrm{C}$ to constant weight. Residual glucose was measured using an SBA-40C bioanalyzer (Institute of Biology, Shandong Academy of Sciences, China). The methods of lipid extraction and fatty acid methyl esters (FAMEs) preparation as previously reported [13]. FAMEs samples were analyzed by gas chromatograph system (GC-2010, Shimadzu, Japan), equipped with a capillary column (DB-23, $60 \mathrm{~m} \times 0.22 \mathrm{~mm}$ ) and a flame ionization detector (FID). The injector was maintained at $250{ }^{\circ} \mathrm{C}$ with an inject volume of $1 \mu \mathrm{L}$. Fatty acids were identified by comparison with related external standards (Sigma, USA). The quantities of individual FAMEs were estimated from the peak areas on the chromatogram using nonadecanoic acid (C19:0) (Sigma, USA) as the internal standard. The DHA percentages in total fatty acids could be determined by area normalization method. The sulfite method $\left(\mathrm{Na}_{2} \mathrm{SO}_{3}\right.$ method) is proposed for the measurement of oxygen transfer coefficients $\left(\mathrm{k}_{\mathrm{L}} \mathrm{a}\right)$ [12].

\section{RNA isolation, library construction, and sequencing}

According to the cell growth characteristics, we separately sampled the early lipid accumulation stage at $36 \mathrm{~h}$ under normal and high oxygen supply conditions. Samples comprising $10 \mathrm{~mL}$ were centrifuged at $5000 \times g$ for 10 min, immersed in RNAlock Reagent, and preserved at $-80{ }^{\circ} \mathrm{C}$ until RNA extraction.

Total RNA was extracted using TRIzol reagent (Zoonbio Biotechnology, Nanjing, China) according to the manufacture's protocol. The RNA was treated with RNase-Free DNase set (NEB, Ipswich, MA, CA, USA) to digest any genomic DNA that might be present. The purity and integrity of RNA samples was evaluated using an RNA Lab-On-A-Chip (Caliper Technologies Corp., Mountain View, CA, USA) on Agilent Bioanaylzer 2100 (Agilent Technologies, Palo Alto, CA, USA). Total RNA was sent to Frasergan Genomics Institute (Wuhan, China) for libraries construction and sequencing. PolyA (+) mRNAs were enriched using the Oligo (dT) 25 Magnetic Beads. Then, the mRNAs were fragmented, followed by the synthesis of cDNA using random hexamer-primer for the first-strand cDNA and buffer, dNTPs, RNase H, and DNA polymerase I for the secondstrand cDNA synthesis. The cDNA fragments were then purified and connected with sequencing adapters. The short fragments with a length of $200 \mathrm{bp}$ were selected 
by agarose gel electrophoresis and sequenced on the HiSeq 2000 platform (Illumina, CA, USA).

\section{RNA-seq data analyses}

Clean data were obtained by filtering unknown nucleotides and low-quality sequences from the raw data. After which, SOA Paligner/SOAP2 software was used to map the clean reads to reference sequences [54]. The gene and transcript expression levels were estimated and normalized using the RPKM method [55], which enables the removal of the influence of different gene length and sequencing discrepancies on the calculation of gene expression levels. Using a rigorous algorithm that was described by Audic and Claverie to identified differentially expressed genes (DEGs) between two samples [56], false discovery rate (FDR) of the results was controlled using Benjamini's and Hochberg's approach. We used an absolute value of the $\log _{2}$ ratio of $\geq 1$ and a FDR of $\leq 0.001$ as criteria to judge the DEGs [57]. For functional enrichment (e.g., Gene ontology (GO) and KEGG), the DEGs were mapped to terms in GO and KEGG database (http://www.geneontology.org/; http://www.genom e.jp/kegg). GO terms of which corrected $p$ value less than 0.05 were defined as significantly enriched GO terms in DEGs. For pathway enrichment analysis, pathways with $Q$ value $\leq 0.05$ are significantly enriched in DEGs.

\section{Quantitative PCR (qPCR) validation}

The qPCR primers (Additional file 1: Table S2) were designed using Primer Premier 5.0 software. A Rapid fungal RNA extraction kit (Zoonbio Biotechnology, Nanjing, China) was used for the qPCR experiment as previously described [58]. The relative expression values were calculated using the $2^{-\Delta \Delta \mathrm{Ct}}$ method [59].

\section{Additional file}

Additional file 1: Figure S1. Summary of draft reads of samples by Illumina deep sequencing. (A) Distribution of number of unique reads. (B) Global comparison of normal-oxygen sample and high oxygen sample by Venn diagrams. (C) Number of upregulated and down-regulated DEGs of the normal-oxygen and high oxygen samples. Figure S2. Gene ontology $(\mathrm{GO})$ functional analysis of unique sequences from normal and high oxygen transcriptome. Unique sequences were assigned to three categories: molecular functional, cellular components, and biological process. Figure S3. KEGG pathway enrichment of assembled unigenes. Rich Factor: the number of DEGs in specific pathway term/the number of all genes in specific pathway term. Gene Number: the number of DEGs in specific pathway. Q value: False discovering rate. Pathways with $Q$ value $\leq 0.05$ are significantly enriched in DEGs. Figure S4. Real-time quantitative PCR results for the FAS, ORFA, ORFB, ORFC, ACC, and ME genes from the Schizochytrium sp. HX-308. Values and error bars represent the means and the standard deviations of triplicate experiments. Table S1. The gene information list mentioned in this paper. Table S2. Primers for genes validated by Quantitative real-time PCR ( $q P C R$ ).
Abbreviations

DHA: docosahexaenoic acid; PUFAs: polyunsaturated fatty acids; FAS: fatty acid synthase; FAS: polyketide synthase; CDW: cell dry weight; TFAs: total fatty acids; DEGs: differently expressed genes; EMP: glycolysis; PPP: pentose phosphate pathway; TCA: tricarboxylic acid; HK: hexokinase; PFK: phosphofructokinase; FBA: fructose-bisphosphate aldolase; PK: pyruvate kinase; G6PD: glucose6-phosphate dehydrogenase; 6PGD: 6-phosphogluconate dehydrogenase; CS: citrate synthase; IDH: isocitrate dehydrogenase; ME: malic enzyme; $\mathrm{ACL}$ : cirate lyase; SOD: superoxide dismutase; CAT: catalase; APX: ascorbate peroxidase; ROS: reactive oxygen species; ACC: acetyl-CoA carboxylase; GSH-PX: glutathione peroxidase.

\section{Authors' contributions}

LJR and $\mathrm{HH}$ conceived the study. ZQB performed the transcriptomic data analysis and wrote the paper. XCH participated in fermentation experiments. XMS and SYZ extracted the RNA of Schizochytrium sp. LJR and XJJ contributed to manuscript revision and approved the final version. All authors read and approved the final manuscript.

\section{Author details \\ ${ }^{1}$ Jiangsu National Synergetic Innovation Center for Advanced Materi- als (SICAM), No. 30 South Puzhu Road, Nanjing 211816, People's Republic of China. ${ }^{2}$ College of Biotechnology and Pharmaceutical Engineering, Nanjing Tech University, No. 30 South Puzhu Road, Nanjing 211816, People's Republic of China. ${ }^{3}$ School of Pharmaceutical Sciences, Nanjing Tech University, No. 30 South Puzhu Road, Nanjing 211816, People's Republic of China. ${ }^{4}$ State Key Laboratory of Materials-Oriented Chemical Engineering, Nanjing Tech Univer- sity, No. 5 Xinmofan Road, Nanjing 210009, People's Republic of China.}

\section{Acknowledgements}

Not applicable.

\section{Competing interests}

The authors declare that they have no competing interests.

Availability of supporting data

The authors promise the availability of supporting data.

\section{Consent for publication}

The authors have consented for publication.

\section{Ethics approval and consent to participate}

Not applicable.

\section{Funding}

This work was financially supported by the Outstanding Youth Foundation of Jiangsu Nature Science Foundation (BK20160092), the Program for Innovative Research Teams in Universities of Jiangsu Province (2015), the Jiangsu Synergetic Innovation Center for Advanced Bio-Manufacture (XTE1829), and General Program on Natural Science Research Project of Higher Education of Jiangsu (18KJB530007).

\section{Publisher's Note}

Springer Nature remains neutral with regard to jurisdictional claims in published maps and institutional affiliations.

Received: 18 April 2018 Accepted: 6 September 2018

Published online: 17 September 2018

\section{References}

1. Uauy R, Dangour AD. Nutrition in brain development and aging: role of essential fatty acids. Nutr Rev. 2006;64:72-91.

2. Janssen $\mathrm{Cl}$, Kiliaan AJ. Long-chain polyunsaturated fatty acids (LCPUFA) from genesis to senescence: the influence of LCPUFA on neural development, aging, and neurodegeneration. Prog Lipid Res. 2014;53:1-17.

3. Costa LG, Manzo L. Contaminants in fish: risk-benefit considerations. Arch Ind Hyg Toxicol. 2007;58(3):367-74. 
4. Jiang Y, Fan KW, Wong RT, Chen F. Fatty acid composition and squalene content of the marine microalga Schizochytrium mangrovei. J Agric Food Chem. 2004;52:1196-200.

5. Panchal BM, Padul MV, Kachole MS. Optimization of biodiesel from dried biomass of Schizochytrium limacinum using methanesulfonic acid-DMC. Renew Energy. 2016;86:1069-74.

6. Ethier S, Woisard K, Vaughan D, Wen ZY. Continuous culture of the microalgae Schizochytrium limacinum on biodiesel-derived crude glycerol for producing docosahexaenoic acid. Bioresour Technol. 2011:102:88-93.

7. Johnson MB, Wen Z. Production of biodiesel fuel from the microalga Schizochytrium limacinum by direct transesterification of algal biomass. Energy Fuels. 2009;23(10):5179-83.

8. Bailey R B, Dimasi D, Hansen J M, et al. Enhanced production of lipids containing polyenoic fatty acid by very high density cultures of eukaryotic microbes infermentors. US Patent 6607900

9. Chang GF, Wu J, Jiang CH, Tian G, Wu Q, Chang M, Wang X. The relationship of oxygen uptake rate and $k_{L}$ a with rheological properties in high cell density cultivation of docosahexaenoic acid by Schizochytrium sp. S31. Bioresour Technol. 2014;152:234-40.

10. Jakobsen AN, Aasen IM, Josefsen KD, Strom AR. Accumulation of docosahexaenoic acid-rich lipid in thraustochytrid Aurantiochytrium sp strain T66: effects of $\mathrm{N}$ and $\mathrm{P}$ starvation and O-2 limitation. Appl Microbiol Biotechnol. 2008;80:297-306.

11. Chi Z, Liu Y, Frear C, Chen S. Study of a two-stage growth of DHA-producing marine algae Schizochytrium limacinum SR21 with shifting dissolved oxygen level. Appl Microbiol Biotechnol. 2009:81:1141-8.

12. Qu L, Ji XJ, Ren LJ, Nie ZK, Feng Y, Wu WJ, et al. Enhancement of docosahexaenoic acid production by Schizochytrium sp. using a two-stage oxygen supply control strategy based on oxygen transfer coefficient. Lett Appl Microbiol. 2011;52:22-7.

13. Ren $\sqcup$, Ji $X J$, Huang $H$, Qu L, Feng $Y$, Tong QQ, et al. Development of a stepwise aeration control strategy for efficient docosahexaenoic acid production by Schizochytrium sp. Appl Microbiol Biotechnol. 2010;87:1649-56.

14. Leaver MJ, Villeneuve LA, Obach A, Jensen L, Bron JE, Tocher DR, Taggart $J B$. Functional genomics reveals increases in cholesterol biosynthetic genes and highly unsaturated fatty acid biosynthesis after dietary substitution of fish oil with vegetable oils in Atlantic salmon (Salmo salar). Bmc Genomics. 2008;9:299.

15. Zhou X, Zhou J, Tian H, Yuan Y. Dynamic lipidomic insights into the adaptive responses of Saccharomyces cerevisiae to the repeated vacuum fermentation. OMICS. 2010;14:563-74.

16. Tang X, Zan X, Zhao L, Chen H, Chen YQ, Chen W, et al. Proteomics analysis of high lipid-producing strain Mucor circinelloides, WJ11: an explanation for the mechanism of lipid accumulation at the proteomic level. Microb Cell Fact. 2016;15:1-16.

17. Zhang W, Li F, Nie L. Integrating multiple 'omics' analysis for microbial biology: application and methodologies. Microbiology. 2010;156(Pt 2):287-301.

18. Metz JG, Roessler P, Facciotti D, Levering C, Dittrich F, Lassner M, et al. Production of polyunsaturated fatty acids by polyketide synthases in both prokaryotes and eukaryotes. Science. 2001;293:290-3.

19. Ye C, Qiao WH, Yu XB, XJ J, Huang H, et al. Reconstruction and analysis of the genome-scale metabolic model of Schizochytrium limacinum SR21 for docosahexaenoic acid production. Bmc Genomics. 2015;16(1):799.

20. Li J, Ren LJ, Sun GN, Qu L, Huang H. Comparative metabolomics analysis of docosahexaenoic acid fermentation processes by Schizochytrium sp. under different oxygen availability conditions. OMICS. 2013;17:269-81.

21. Ma ZX, Tan YZ, Cui GZ, Feng YG, Cui Q, Song XJ. Transcriptome and gene expression analysis of DHA producer Aurantiochytrium under low temperature conditions. Sci Rep. 2015;5:14446.

22. Morin N, Cescut J, Beopoulos A, Lelandais G, Berre VL, Uribelarrea JL, et al. Transcriptomic Analyses during the Transition from Biomass Production to Lipid Accumulation in the Oleaginous Yeast Yarrowia lipolytica. PLoS ONE. 2011;6(11):e27966.

23. Chen H, Hao G, Wang L, Wang H, Gu Z, Liu L, et al. Identification of a critical determinant that enables efficient fatty acid synthesis in oleaginous fungi. Sci Rep. 2015;5:11247.

24. Ji XJ, Mo KQ, Ren LJ, Li GL, Huang H. Genome sequence of Schizochytrium sp. CCTCC M209059, an effective producer of docosahexaenoic acid-rich lipids. Genome Announc. 2015;3(4):e00819.
25. Gessler NN, Aver'Yanov AA, Belozerskaya TA. Reactive oxygen species in regulation of fungal development. Biochemistry. 2007;72(10):1091-109.

26. Ren $L$, Hu XC, Zhao XY, Chen SL, Wu Y, Li D, et al. Transcriptomic analysis of the regulation of lipid fraction migration and fatty acid biosynthesis in Schizochytrium sp. Sci Rep. 2017;7(1):3562.

27. Lin Z, Zhao H, Lai Y, Wu J, Chen H. Improving docosahexaenoic acid productivity of Schizochytrium sp. by a two-stage AEMR/shake mixed culture mode. Bioresour Technol. 2013;142:719-22.

28. Zhao X, Ren L, Guo D, Wu W, Ji X, Huang H. CFD investigation of Schizochytrium sp. impeller configurations on cell growth and docosahexaenoic acid synthesis. Bioprocess Biosyst Eng. 2016;39:1297-304.

29. Sun XM, Ren LJ, Ji XJ, Chen SL, Guo DS, Huang H. Adaptive evolution of Schizochytrium sp. by continuous high oxygen stimulations to enhance docosahexaenoic acid synthesis. Bioresour Technol. 2016;211:374-81.

30. Valledor L, Furuhashi T, Hanak AM, Weckwerth W. Systemic cold stress adaptation of Chlamydomonas reinhardtii. Mol Cell Proteom. 2013;12:2032-47.

31. Godon $\mathrm{C}$, Lagniel $\mathrm{G}$, Lee J, Buhler JM, kieffer S, Perrot M, et al. The $\mathrm{H}_{2} \mathrm{O}_{2}$ stimulon in Saccharomyces cerevisiae. J Biol Chem. 1998;273:22480-9.

32. Cheng RL, Feng J, Zhang BX, Huang Y, Cheng J, Zhang CX. Transcriptome and gene expression analysis of an oleaginous diatom under different salinity conditions. Bioenergy Res. 2014;7:192-205.

33. Osada K, Maeda Y, Yoshino T, Nojma D, Bowler C, Tanaka T. Enhanced $\mathrm{NADPH}$ production in the pentose phosphate pathway accelerates lipid accumulation in the oleaginous diatom Fistulifera solaris. Algal Res. 2017:23:126-34.

34. Wynn JP, Abdul Hamid A, Ratledge C. The role of malic enzyme in the regulation of lipid accumulation in filamentous fungi. Microbiology. 1999:145:1911-7.

35. Zhang Y, Adams IP, Ratledge C. Malic enzyme: the controlling activity for lipid production? Overexpression of malic enzyme in Mucor circinelloides leads to a 2.5-fold increase in lipid accumulation. Microbiology. 2007;153:2013-25.

36. Ratledge C. Fatty acid biosynthesis in microorganisms being used for single cell oil production. Biochimie. 2004;86(11):807-15.

37. RzezniczakTZ, Merritt TJS. Interactions of NADP-reducing enzymes across varying environmental conditions: a model of biological complexity. G3 (Bethesda). 2012;2(12):1613-23.

38. Lehmann M, Schwarzlaender M, Obata T, Sirikantaramas S, Burow M, Olsen $C E$, et al. The metabolic response of arabidopsis roots to oxidative stress is distinct from that of heterotrophic cells in culture and highlights a complex relationship between the levels of transcripts, metabolites, and flux. Mol Plant. 2009;2(3):390-406.

39. Yan J, Cheng R, Lin X, You S, Li K, Rong H, Ma Y. Overexpression of acetylCoA synthetase increased the biomass and fatty acid proportion in microalga Schizochytrium. Appl Microbiol Biotechnol. 2013;97:1933-9.

40. Harris RA, Joshi M, Jeoung NH, Obayashi M. Overview of the molecular and biochemical basis of branched-chain amino acid catabolism. J Nutr. 2005;135(6 Suppl):1527S-30S

41. Pietrocola F, Galluzzi L, Bravo-Sanpedro J, Madeo F, Kroemer G. Acetyl coenzyme a: a central metabolite and second messenger. Cell Metab. 2015;21(6):805-21.

42. Chen W, Zhou PP, Zhang M, Zhu YM, Wang XP, Luo XA, et al. Transcriptome analysis reveals that up-regulation of the fatty acid synthase gene promotes the accumulation of docosahexaenoic acid in Schizochytrium sp. S056 when glycerol is used. Algal Res. 2016;15:83-92.

43. Napier JA. Plumbing the depths of PUFA biosynthesis: a novel polyketide synthase-like pathway from marine organisms. Trends Plant Sci. 2002; 7:51-4.

44. Lippmeier JC, Crawford KS, Owen CB, Rivas AA, Metz JG, Apt KE. Characterization of both polyunsaturated fatty acid biosynthetic pathways in Schizochytrium sp. Lipids. 2009:44:621-30.

45. Hoang MH, Nguyen C, Pham HQ, Nauyen LV, Le HD, et al. Transcriptome sequencing and comparative analysis of Schizochytrium mangrovei, PQ6 at different cultivation times. Biotech Lett. 2016;38(10):1-9.

46. Ratledge C, Wynn JP. The biochemistry and molecular biology of lipid accumulation in oleaginous microorganisms. Adv Appl Microbiol. 2002;51(1-44):1-51.

47. Guichardant M, Chen P, Liu M, Calzada C, Colas R, Vericel E, et al. Functional lipidomics of oxidized products from polyunsaturated fatty acids. Chem Phys Lipids. 2011;164:544-8. 
48. Johansson M, Chen X, Milanova S, Santos C, Petranovic D. PUFA-induced cell death is mediated by Yca1 p-dependent and -independent pathways, and is reduced by vitamin C in yeast. Fems Yeast Res. 2016;16(2):007.

49. Ruenwai R, Neiss A, Laoteng K, Vongsangnak W, Dalfard AB, Cheevadhanarak S, et al. Heterologous production of polyunsaturated fatty acids in Saccharomyces cerevisiae causes a global transcriptional response resulting in reduced proteasomal activity and increased oxidative stress. Biotechnol J. 2015;6:343-56.

50. Tripathi BN, Gaur JP. Relationship between copper- and zinc-induced oxidative stress and proline accumulation in Scenedesmus sp. Planta. 2004;219:397-404.

51. Turrens JF. Superoxide production by the mitochondrial respiratory chain. Biosci Rep. 1997;17:3-8.

52. Kumar J, Singh VP, Prasad SM. NaCl-induced physiological and biochemical changes in two cyanobacteria Nostoc muscorum and Phormidium foveolarum acclimatized to different photosynthetically active radiation. $J$ Photochem Photobiol B. 2015;151:221-32.

53. Li Q, McNeil B, Harvey LM. Adaptive response to oxidative stress in the filamentous fungus Aspergillus niger B1-D. Free Rad Biol Med. 2008;44:394-402.
54. Li R, Yu C, Li Y, Lam TW, Yiu SM, Kristiansen K, Wang J. SOAP2: an improved ultrafast tool for short read alignment. Bioinformatics. 1966;25:1966-7.

55. Mortazavi A, Williams BA, Mccue K, Schaeffer L, Wold B. Mapping and quantifying mammalian transcriptomes by RNA-Seq. Nat Methods. 2008;5:621-8.

56. Audic S, Claverie JM. The significance of digital gene expression profiles. Genome Res. 1997;7:986-95.

57. Benjamini Y, Yekutieli D. The control of the false discovery rate in multiple testing under dependency. Ann Stat. 2001;29(4):1165-88.

58. Zhu Y, Zhou P, Hu J, Zhang R, Ren L, Li M, Ning F, Chen W, Yu L. Characterization of Pythium transcriptome and gene expression analysis at different stages of fermentation. PLoS ONE. 2013;8:e65552.

59. Livak KJ, Schmittgen TD. Analysis of relative gene expression data using real-time quantitative PCR and the $2^{-\triangle \Delta C T}$ method. Methods. 2001;25:402-8.
Ready to submit your research? Choose BMC and benefit from:

- fast, convenient online submission

- thorough peer review by experienced researchers in your field

- rapid publication on acceptance

- support for research data, including large and complex data types

- gold Open Access which fosters wider collaboration and increased citations

- maximum visibility for your research: over $100 \mathrm{M}$ website views per year

At BMC, research is always in progress.

Learn more biomedcentral.com/submissions 